\title{
AN INTEGRALITY THEOREM OF GROSSHANS OVER ARBITRARY BASE RING
}

\author{
WILBERD VAN DER KALLEN \\ Mathematisch Instituut \\ P.O. Box 80.010 \\ 3508 TA Utrecht \\ The Netherlands \\ W.vanderKallen@uu.nl
}

Dedicated to the memory of T. A. Springer

\begin{abstract}
We revisit a theorem of Grosshans and show that it holds over arbitrary commutative base ring k. One considers a split reductive group scheme $G$ acting on a k-algebra $A$ and leaving invariant a subalgebra $R$. Let $U$ be the unipotent radical of a split Borel subgroup scheme. If $R^{U}=A^{U}$ then the conclusion is that $A$ is integral over $R$.
\end{abstract}

\section{Introduction}

In G92 Grosshans considered a reductive algebraic group $G$ defined over an algebraically closed field $\mathbf{k}$ acting algebraically on a commutative $\mathbf{k}$-algebra $A$. Fix a Borel subgroup $B$ with unipotent radical $U$. Then Grosshans considered the smallest $G$-invariant k-subalgebra $G \cdot A^{U}$ of $A$ that contains the fixed point algebra $A^{U}$. He showed that $A$ is integral over $G \cdot A^{U}$. If $R$ is any other $G$-invariant k-subalgebra of $A$ that contains $A^{U}$ it then follows that $A$ is integral over $R$. One of the tools used by Grosshans is what is called power reductivity in FvdK]. As it is shown in $[\mathrm{FvdK}]$ that power reductivity holds over arbitrary commutative base ring $\mathbf{k}$, we now set out to prove the integrality result of Grosshans in the same generality. We need a little care as we are not even assuming that the ground ring is noetherian.

\section{Preliminaries}

We use an arbitrary commutative ring $\mathbf{k}$ as base ring. Let $A$ be a commutative k-algebra. We say that an affine algebraic group scheme $G$ acts on $A$ if $A$ is a $G$-module [J] and the multiplication map $A \otimes_{\mathbf{k}} A \rightarrow A$ is a $G$-module map. Then the coaction $A \rightarrow A \otimes_{\mathbf{k}} \mathbf{k}[G]$ is an algebra homomorphism. One also says that $G$ acts rationally on $A$ by algebra automorphisms. Geometrically it means that $G$ acts from the right on $\operatorname{Spec} A$.

Received . Accepted . 
Lemma 1. Let $G$ be a smooth affine algebraic group scheme over $\mathbf{k}$. Let $G$ act on the commutative $\mathbf{k}$-algebra $A$. Then the nilradical of $A$ is a $G$-submodule.

Proof. (Thanks to Angelo Vistoli http://mathoverflow.net/questions/68366/ for explaining to me that smoothness is the right condition.)

As the base change map $A_{\text {red }} \rightarrow A_{\text {red }} \otimes_{\mathbf{k}} \mathbf{k}[G]$ is a smooth map, $A_{\text {red }} \otimes_{\mathbf{k}} \mathbf{k}[G]$ is reduced, by [EGA4, Prop. (17.5.7)] or by [stacks, Lemma 033B] with URL http://stacks.math. columbia.edu/tag/033B.

Now let $N$ denote the nilradical of $A$. The coaction $A \rightarrow A \otimes \mathbf{k}[G]$ sends $N$ to the nilradical $N \otimes_{\mathbf{k}} \mathbf{k}[G]$ of $A \otimes_{\mathbf{k}} \mathbf{k}[G]$.

From now on let $G=G_{\mathbf{k}}$, where $G_{\mathbb{Z}}$ is a Chevalley group over $\mathbb{Z}$. In other words, $G$ is a split reductive group scheme over k under the conventions of SGA3. Choose a split maximal torus $T$, a standard Borel subgroup $B$ and its unipotent radical $U$.

Lemma 2. The coordinate ring $\mathbf{k}[G]$ is a free $\mathbf{k}$-module.

Proof. As $\mathbf{k}[G]=\mathbb{Z}\left[G_{\mathbb{Z}}\right] \otimes_{\mathbb{Z}} \mathbf{k}$ it suffices to treat the case $\mathbf{k}=\mathbb{Z}$. Now the coordinate ring of $G$ is a subring of the coordinate ring of the big cell. And the coordinate ring of the big cell is clearly free as a $\mathbb{Z}$-module. Now use that a submodule of a free $\mathbb{Z}$-module is free [HS, Chapter I, Theorem 5.1].

Lemma 3. If $V$ is a $G$-module and $v \in V$, then the $G$-submodule generated by $v$ exists and is finitely generated as a $\mathbf{k}$-module.

Proof. As $\mathbf{k}[G]$ is a free $\mathbf{k}$-module, this follows from [SGA3, Exposé VI, Lemme 11.8].

See also [S, Proposition 3]. Note that the existence result in the Lemma does not follow from the fact that $G$ is flat over k SGA3, Exposé VI, Édition 2011, Remarque 11.10.1].

Definition 1. Recall that we call a homomorphism of k-algebras $f: A \rightarrow B$ power surjective [FvdK, Definition 2.1] if for every $b \in B$ there is an $n \geq 1$ so that the power $b^{n}$ is in the image of $f$.

A flat affine group scheme $H$ over $\mathbf{k}$ is called power reductive [FvdK, Definition 2] if the following holds.

Property (Power Reductivity). Let $L$ be a cyclic k-module with trivial $H$ action. Let $M$ be a rational $H$-module, and let $\varphi$ be an $H$-module map from $M$ onto $L$. Then there is a positive integer $d$ such that the $d$-th symmetric power of $\varphi$ induces a surjection:

$$
\left(S^{d} M\right)^{H} \rightarrow S^{d} L .
$$

Here $V^{H}=H^{0}(H, V)$ denotes the submodule of invariants in an $H$-module $V$.

Proposition 4. Let $H$ be a flat affine algebraic group scheme over $\mathbf{k}$. The following are equivalent

(1) $H$ is power reductive,

(2) for every power surjective $H$-homomorphism of commutative $\mathbf{k}$-algebras $f: A \rightarrow B$ the map $A^{H} \rightarrow B^{H}$ is power surjective. 
Proof. First assume 1. Let $f: A \rightarrow B$ be power surjective and let $b \in B^{H}$. Choose $n \geq 1$ so that $b^{n} \in f(A)$. Let $M=f^{-1}(L)$ be the inverse image of $L=\mathbf{k} b^{n}$. Choose $d \geq 1$ so that $\left(S^{d} M\right)^{H} \rightarrow S^{d} L$ is surjective. Multiplication induces $H$-module maps $S^{d} A \rightarrow A, S^{d} B \rightarrow B$. One has a commutative diagram of $H$-homomorphisms

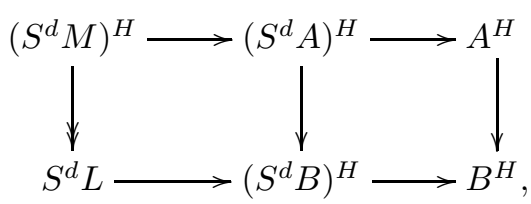

and one sees that $b^{n d}$ lies in the image of $A^{H}$ because it lies in the image of $S^{d} L$.

Conversely, assume 2 and let $M \rightarrow L$ be given as in the Property. One has a surjective map of symmetric algebras $S^{*}(M) \rightarrow S^{*}(L)$. Now let $b$ be a generator of $S^{1} L$. There is a power $b^{d} \in S^{d} L$ of $b$ that lies in the image of $\left(S^{*}(M)\right)^{H}$. But then $\left(S^{d} M\right)^{H} \rightarrow S^{d} L$ is surjective.

Remark 1. So the finite generation hypothesis does not belong in FvdK, Proposition 6]. Note that there is no finiteness hypothesis on $M$ in the Power Reductivity Property.

Proposition 5. Let $G$ act rationally by $\mathbf{k}$-algebra automorphisms on the commutative algebra $A$ and let $J$ be a $G$-invariant ideal. Then $A^{U} \rightarrow(A / J)^{U}$ is power surjective.

Proof. The transfer principle [G97, Ch. Two] tells that $A^{U}=\left(A \otimes_{\mathbf{k}} \mathbf{k}[G / U]\right)^{G}$, where $\mathbf{k}[G / U]$ means the algebra of $U$-invariants in $\mathbf{k}[G]$ under the action by right translation. Here is one proof. Write

$$
A^{U}=\operatorname{hom}_{U}(\mathbf{k}, A)=\operatorname{hom}_{G}\left(\mathbf{k}, \operatorname{ind}_{U}^{G} A\right)=\left(\operatorname{ind}_{B}^{G} \operatorname{ind}_{U}^{B} A\right)^{G}=\left(\operatorname{ind}_{B}^{G}\left(A \otimes_{\mathbf{k}} \mathbf{k}[T]\right)\right)^{G},
$$

where the $B$-module $\mathbf{k}[T]$ is a direct sum of weights of $B$, so that $\operatorname{ind}_{B}^{G}\left(A \otimes_{\mathbf{k}} \mathbf{k}[T]\right)$ equals $A \otimes_{\mathbf{k}} \operatorname{ind}_{B}^{G}(\mathbf{k}[T])$ by the tensor identity for weights [FvdK, Proposition 17]. Further $\operatorname{ind}_{B}^{G} \mathbf{k}[T]=\operatorname{ind}_{U}^{G} \mathbf{k}=\mathbf{k}[G / U]$, so $A^{U}=\left(A \otimes_{\mathbf{k}} \mathbf{k}[G / U]\right)^{G}$ indeed. We may identify $A^{U} \rightarrow(A / J)^{U}$ with $\left(A \otimes_{\mathbf{k}} \mathbf{k}[G / U]\right)^{G} \rightarrow\left(A / J \otimes_{\mathbf{k}} \mathbf{k}[G / U]\right)^{G}$. Now use that $G$ is power reductive [FvdK, Theorem 12] and apply Proposition 4 ,

\section{The integrality theorem}

If $G$ acts rationally by k-algebra automorphisms on our algebra $A$, we denote by $G \cdot A^{U}$ the k-subalgebra generated by the $G$-submodules generated by the elements of $A^{U}$. Thus $G \cdot A^{U}$ is the smallest $G$-invariant subalgebra of $A$ that contains $A^{U}$. Our main result is the following generalization of [G92, Theorem 5].

Theorem 6. The algebra $A$ is integral over $G \cdot A^{U}$.

Proof. As Grosshans works over an algebraically closed field there are some details that need to be checked now. Let $v \in A$. We have to show that $v$ is integral over $G \cdot A^{U}$. Let $V$ be the $G$-submodule of $A$ generated by $v$ and consider the 
symmetric algebra $S_{\mathbf{k}}^{*}(V)$ on $V$. Using the obvious map from $S_{\mathbf{k}}^{*}(V)$ to $A$ one sees that it suffices to prove the theorem for the algebra $S_{\mathbf{k}}^{*}(V)$. So from now on let $A=S_{\mathbf{k}}^{*}(V)$. Let $A^{+}$be the augmentation ideal generated by $V$ in $A$. Let $J$ be the ideal of $A$ generated by $A^{+} \cap\left(G \cdot A^{U}\right)$. It is $G$-invariant, so its radical $\sqrt{J}$ is also $G$-invariant, by lemma 1 applied to $A / J$. We claim that $A^{+} \subseteq \sqrt{J}$. Suppose not. Then $A^{+} /\left(A^{+} \cap \sqrt{J}\right)$ is nontrivial. Now every nontrivial $G$-module $N$ has at least one nontrivial $U$-invariant. (Note that by lemma 3 we may reduce to the case that $N$ has finitely many weight spaces.) Say $0 \neq f \in\left(A^{+} /\left(A^{+} \cap \sqrt{J}\right)\right)^{U}$. View $f$ as a nonzero element of $A / \sqrt{J}$. By Proposition 5 there is a power of $f$ that lies in the image of $A^{U}$ in the algebra $A / \sqrt{J}$. But then it actually lies in the image of $A^{+} \cap\left(G \cdot A^{U}\right)$, hence in the image of $J$, which is zero. But $A / \sqrt{J}$ is reduced; contradiction.

Let $v_{1}, \ldots, v_{n}$ generate $V$ as a k-module. Every element $f$ of $J$ may be written as a sum of terms $a_{I} v^{I}$, where $I=\left(I_{1}, \ldots, I_{n}\right), a_{I} \in A^{+} \cap\left(G \cdot A^{U}\right)$ and $v^{I}:=v_{1}^{I_{1}} \cdots v_{n}^{I_{n}}$. Moreover, if $f$ is homogeneous of degree $d$, then the $a_{I}$ may be taken homogeneous of degree $d-|I|$ where $|I|=I_{1}+\cdots+I_{n}$. In particular, all terms have $|I|<d$. As $A^{+} \subseteq \sqrt{J}$ we may choose $m$ so large that $v_{i}^{m} \in J$ for all $i$. So then $v_{i}^{m}$ may be written as a sum of terms $a_{I} v^{I}$ with $|I|<m$. It follows that $A^{+}$is generated as a $G \cdot A^{U}$-module by finitely many $v^{I}$. The theorem follows.

Let $B^{\text {opp }}$ denote the Borel subgroup scheme containing $T$ that is opposite to $B$. If $V$ is a $T$-module, then $\inf _{T}^{B^{\text {opp }}} V$ denotes the $B^{\text {opp }}$-module obtained by composition with the standard homomorphism $B^{\mathrm{opp}} \rightarrow T$. Recall that Grosshans has introduced a filtration on any $G$-module $M$ (after Luna). Its associated graded module $\operatorname{gr} M$ can be embedded into the module hull $\nabla(\operatorname{gr} M):=\operatorname{ind}_{B^{\text {opp }}}^{G} \inf _{T}^{B^{\text {opp }}} M^{U}$. One knows that $(\text { hull } \nabla(\operatorname{gr} M))^{U}=(\operatorname{gr} M)^{U}$ and that $\mathrm{H}^{i}(G$, hull $\nabla(\operatorname{gr} M))$ vanishes for positive $i$. If $G$ acts on the commutative k-algebra $A$, then $\operatorname{gr} A$ and $\operatorname{hull}_{\nabla}(\operatorname{gr} A)$ are commutative $\mathbf{k}$-algebras. See $\mathrm{FvdK}$ for details on all this.

We now get a proof of [FvdK, Theorem 32] in the style of Grosshans [G92].

Corollary 7. Let $A$ be a finitely generated commutative $\mathbf{k - a l g e b r a ~ o n ~ w h i c h ~} G$ acts rationally by $\mathbf{k}$-algebra automorphisms. If $\mathbf{k}$ is Noetherian, there is a positive integer $n$ so that:

$$
n \operatorname{hull}_{\nabla}(\operatorname{gr} A) \subseteq \operatorname{gr} A .
$$

In particular $\mathrm{H}^{i}(G, \operatorname{gr} A)$ is annihilated by $n$ for positive $i$.

Proof. As in the proof of [G92, Theorem 8] theorem 6] shows that hull $\nabla(\operatorname{gr} A)$ is integral over gr $A$. As it is also a finitely generated k-algebra $\mathrm{FvdK}$, Theorem $30]$, it is a finitely generated module over $\operatorname{gr} A$. View hull $\nabla(\operatorname{gr} A) \otimes_{\mathbb{Z}} \mathbb{Q}$ as a $G_{\mathbb{Q}^{-}}$ module [FvdK, Remark 52]. It is a direct sum of modules $\operatorname{ind}_{B^{\text {opp }}}^{G} \inf _{T}^{B^{\text {opp }}} A_{\lambda}^{U} \otimes_{\mathbb{Z}} \mathbb{Q}$ with highest weight $\lambda$ (if we consider the roots of $B$ positive). As the image of gr $A \otimes_{\mathbb{Z}} \mathbb{Q}$ in hull $\nabla$ (gr $\left.A\right) \otimes_{\mathbb{Z}} \mathbb{Q}$ contains the highest weight spaces, the injection $\operatorname{gr} A \rightarrow \operatorname{hull}_{\nabla}(\operatorname{gr} A)$ becomes an isomorphism after tensoring with $\mathbb{Q}$. So hull $_{\nabla}(\operatorname{gr} A) / \operatorname{gr} A$ is a finitely generated gr $A$-module and a torsion abelian group. Choose $n>0$ so that $n$ annihilates $\operatorname{hull}_{\nabla}(\operatorname{gr} A) / \operatorname{gr} A$. Then it also annihilates $\mathrm{H}^{i-1}\left(G, \operatorname{hull}_{\nabla}(\operatorname{gr} A) / \operatorname{gr} A\right)$, hence $\mathrm{H}^{i}(G, \operatorname{gr} A)$, for $i>0$. 


\section{References}

[FvdK] Vincent Franjou and Wilberd van der Kallen, Power reductivity over an arbitrary base, Documenta Mathematica, Extra Volume Suslin (2010), 171-195.

[G92] Frank D. Grosshans, Contractions of the actions of reductive algebraic groups in arbitrary characteristic, Invent. Math. 107 (1992), 127-133.

[G97] Frank D. Grosshans, Algebraic homogeneous spaces and invariant theory, Lecture Notes in Mathematics 1673, Springer-Verlag, Berlin, 1997.

[HS] P.J. Hilton and U. Stammbach, A Course in Homological Algebra, Graduate Texts in Mathematics 4, Springer-Verlag, 1971.

[J] Jens Carsten Jantzen, Representations of algebraic groups, Second edition. Mathematical Surveys and Monographs 107, American Mathematical Society, Providence, RI, 2003.

[EGA4] Alexandre Grothendieck, Éléments de géométrie algébrique, (rédigés avec la collaboration de Jean Dieudonné) : IV. Étude cohomologique des faisceaux cohérents, Quatrième partie, Publications Mathématiques de l'IHÉS 32 (1967), 5-361.

[SGA3] M. Demazure, A. Grothendieck, Schémas en groupes I, II, III, Lecture Notes in Math 151, 152, 153, Springer-Verlag, New York (1970) and new edition in Documents Mathématiques 7, 8 of Société Mathématique de France (2011).

[stacks] The Stacks Project Authors, http://stacks.math.columbia.edu

[S] C. S. Seshadri, Geometric reductivity over arbitrary base, Advances in Math. 26 (1977), no. 3, 225-274. 